\title{
Sensitivity of Cytokine and Cytokine Mediator Detection aiding in Diagnosis of Premature Coronary Artery Disease Patients
}

\author{
Wafa Munir Ansari ${ }^{1}$, Dilshad Ahmad Khan ${ }^{1}$, Omer Jamshed Khan ${ }^{2}$ and Farooq Ahmad Khan ${ }^{1}$ \\ ${ }^{1}$ Department of Chemical Pathology, Army Medical College, National University of Science and Technology (NUST), Islamabad, Pakistan \\ ${ }^{2}$ Armed Forces Institute of Rehabilitation Medicine/Cardiac Rehab, Rawalpindi, Pakistan
}

Received: July 14, 2014; Accepted: February 20, 2015; Published: March 04, 2015

*Corresponding author: Wafa Munir Ansari, Department of Chemical Pathology, Army Medical College, National University of Science and technology (NUST), Islamabad, Pakistan, Tel: +92-03215505342; Fax: +92-051-9271247; E-mail: wafamuniransari@yahoo.com

\section{Abstract}

Introduction: The objective was to evaluate diagnostic accuracy of high sensitivity C-reactive protein (hS CRP), Interleukin-18 (IL-18), Tumor Necrosis Factor-alpha (TNF-alpha), Interleukin-10 (IL- 10), IL-18/IL- 10 ratio and their serum cut off values for identification of Premature Coronary Artery Disease (PCAD) patients.

Method: It was a diagnostic validation case-control study carried out at the Clinical Pathology Laboratories of the Army Medical College, Rawalpindi, Pakistan, from May, 2013 to Jan, 2014. Two hundred and fifty subjects aged $<45$ years scheduled to undergo coronary angiography were consecutively screened. Out of these ninetyeight patients with $>70 \%$ stenosis, in at least one coronary vessel on angiography, were labeled to have PCAD. An equal number of angio-negative subjects were taken as controls from the study group. Serum IL- 10, IL- 18 and TNF alpha were measured using Enzyme Linked Immuno-sorbent Assay (ELISA), hS CRP on Immulite 1000 and serum cholesterol, Triglycerides and High Density Lipoprotein (HDL) by colorimetric methods. Statistical analysis was done using SPSS- 17 and MedCalc software.

Results: Total 196 subjects consisting of 98 PCAD patients and 98 angio-negative controls participated in the study. Mean \pm SD age of PCAD patients was $40.7 \pm 4.23$ yrs ( 89 males and 9 females) while in controls it was $35.1 \pm 7.55$ yrs ( 93 males and 5 females). Serum hS CRP had highest area under curve AUC (95\% confidence interval) of 0.936 (0.89- 0.97) while AUC $(95 \% \mathrm{CI})$ of IL-18, TNF alpha and IL-10 were $0.853(0.79-0.90), 0.731(0.659-0.796)$ and 0.574 (0.4970.649 ) respectively. Sensitivity-specificity of hS CRP and IL-18 at cut off value of $3.18 \mathrm{pg} / \mathrm{ml}$ and $200 \mathrm{pg} / \mathrm{ml}$ were $86 \%-91 \%$ and $77 \%-81 \%$ respectively while that of IL-18/IL-10 ratio and IL-10 at cut off values $>138.9$ and $>0.3 \mathrm{pg} / \mathrm{ml}$ were $68-64 \%$ and $20-100 \%$ respectively. Significant correlation was observed between hS CRP $(p<0.01)$, IL$18(p<0.01)$ and TNF alpha $(p<0.05)$.

Conclusion: hS CRP and IL-18 have the best diagnostic potential among the cytokines for detection of PCAD with high sensitivity and specificity. IL-18/IL-10 ratio had moderate significance in the diagnosis of PCAD although it correlated well with the disease burden. IL-10 had $100 \%$ specificity highlighting its role in the diagnosis of the disease but its low sensitivity is a limiting factor. Thus, hS CRP and IL-18 are promising potential biochemical parameters aiding in the clinical diagnosis of Premature Coronary Artery Disease.

Keywords: Diagnosis; Heart disease; hS CRP; Interleukin- 18; Interleukin- 10; TNF alpha

\section{Abbreviations}

TNF alpha: Tumor Necrosis Factor-alpha; IL-18: Interleukin-18; IL-10: Interleukin-10; hS CRP: High Sensitivity C- Reactive Protein; PCAD: Premature Coronary Artery Disease; AUC: Area under Curve; ROC: Receiver Operator Curves; CV: Coefficient of Variation

\section{Introduction}

Premature Coronary Artery Disease (PCAD) is one of the earliest manifestations of coronary atherosclerosis. Effective management of PCAD therefore requires timely detection and accurate evaluation of these patients. Inflammation is proposed to be the main event in the pathogenesis of atherosclerotic plaque formation and progression in CAD [1]. The inflammatory cascade has counter balancing factors that maintain a delicate balance of pro- and anti-inflammatory molecules that regulate vascular homeostasis and maintain integrity of the vessel wall [2]. Multiple pro-inflammatory and anti-inflammatory cytokines are involved in the pathogenesis of CAD having overlapping, antagonistic and synergistic effects. Interleukin-18 (IL-18), tumor necrosis factor-alpha (TNF alpha), High-sensitivity C-reactive protein (hS CRP) and interleukin-10 (IL-10) are being considered to play an essential role in the modulation of immunological and inflammatory processes during various stages of premature coronary artery disease.

IL-18 induces the expression of pro-inflammatory cytokines and Chemokines such as Interleukin-6 (IL-6), IL-8 and Monocyte Chemo attractant Protein-1 (MCP-1) [3]. TNF alpha is also a proinflammatory cytokine which is up regulated in ischemia inducing activation of xanthine oxidase and production of $\mathrm{O}_{2}{ }^{*-}$, leading to dysfunctional coronary endothelium [4]. Samnegard et al. [5] demonstrated that the systemic concentrations of IL-18 and TNF alpha were higher in post myocardial patients as compared to the controls. Moreover, individuals with elevated levels of TNF alpha were at increased risk for acute myocardial infarction and CAD [6]. High sensitive C-reactive protein (hS CRP) is an established proinflammatory biomarker for the detection of individuals at a risk 
of coronary artery disease [7]. Several studies have also shown that increased levels of fibrinogen, CRP and IL- 6 are associated not only with the risk of coronary artery disease but also with its clinical course, progression and severity [8]. Interleukin-10 (IL10 ) on the other hand is an anti-inflammatory cytokine which is associated with a humoral immune response that acts by limiting the local inflammatory response which provides stability to the atherosclerotic lesion [9]. We sought to assess the role of cytokine mediators IL-18, TNF alpha, hS CRP, IL-10, IL-18/IL-10 ratio and TNF alpha/IL-10 ratio in the diagnosis of PCAD patients.

\section{Methodology}

The diagnostic validation case-control study was conducted at the Clinical Pathology Laboratory (CPL), Army Medical College, Rawalpindi, in collaboration with the National Institute of Heart Diseases (NIHD), Rawalpindi, Pakistan. Ethical approval was duly sought from the Institutional Review Committee of Army Medical College (AM College) under the auspices of the National University of Science and Technology (NUST) Islamabad, Pakistan. Duration of the study was 8 months.

\section{Subjects}

Two hundred and fifty subjects aged $<45$ years scheduled to undergo coronary angiography were consecutively screened. Out of these 196 subjects who gave consent and fulfilled our inclusion criteria participated in the study. These included ninety-eight patients with $>70 \%$ stenosis in at least one coronary vessel on angiography which is the gold standard, were labeled to have PCAD. Those who were angiographically proven to be disease free were labeled as controls $(\mathrm{n}=98)$ after informed consent. Only those patients who had $>70 \%$ stenosis in at least 1 coronary vessel on coronary angiography which is the current gold standard were taken as PCAD patients. Patients with a history of hematological, neoplastic, renal, liver or thyroid disease were excluded. Furthermore, patients with infectious or autoimmune diseases, familial hyperlipidemia, congenital heart disease, hepatitis, valvular heart disease, asthma, rheumatoid arthritis or life expectance of less than 12 months and those unable to give informed consent were also excluded. In the controls subjects with any acute or chronic illness or those on anti- inflammatory drugs were excluded. Pregnant women and women on contraceptives were also excluded from the study. Demographic characteristics including cardiovascular risk factors such as hypertension; diabetes mellitus, dyslipidaemia and smoking were noted. Medical examination by a general physician was conducted.Written informed consent was obtained from subjects.

\section{Biochemical Analysis}

All laboratory investigations were carried out in the Clinical Pathology Laboratories of AM College, NUST Rawalpindi, Pakistan. Blood samples were taken in the morning on the day of angiography of the respective patient. $5 \mathrm{ml}$ blood sample was obtained by venipuncture and transferred to a plain vacutainer tube for serum analysis. Serum was separated by centrifugation at $1500 \mathrm{x}$ g for 15 minutes and stored at $-70^{\circ} \mathrm{C}$ until biochemical analysis.
Enzyme Linked Immuno-sorbent Assay (ELISA) technique was used for measuring the concentrations of serum IL-10, IL18, and TNF alpha using human IL-10, IL-18, and TNF alpha, (Bender med Systems, Austria) commercial kits with monoclonal antibodies. The calculated overall intra assay Coefficient of Variation (CV) for IL-18, TNF alpha and IL-10 was 6.5\%, 6.0\% and $3.2 \%$ respectively while the limit of detection was $9 \mathrm{pg} / \mathrm{ml}, 2.3 \mathrm{pg} /$ $\mathrm{ml}$ and $0.1 \mathrm{pg} / \mathrm{ml}$ respectively. The inter assay CV was $3.4 \%, 3.8 \%$ and $3.0 \%$ for IL-18, TNF alpha and IL-10 respectively. Analysis of hS CRP was done by a chemiluminescent immunoassay on Immulite 1000 using kit provided by Siemens (UK). The interassay coefficient of variation (CV) was 3.5\%. Serum cholesterol was measured by cholesterol oxidase method (CHOD-POD) and serum triglyceride was measured by GPO-POD Colorimetric method. All the analytes were run on Selectra E (Vital Scientific, Netherland) using kits provided by Pioneer Diagnostics (USA). $\mathrm{CV}$ of the method was $<1 \%$.

Coronary angiography was performed by trained cardiologists by Jut kin technique using a quantitative coronary angiographic system. Degree of atherosclerosis was calculated using the Gensini score [10]. All coronary angiograms were evaluated by the cardiologists who are unaware of the biochemical analysis results to avoid bias.

\section{Statistical Analysis}

Statistical analysis was performed using SPSS- 17 (SPSS Inc, Chicago) and MedCalc software version 9.6.4.0. KolmogorovSmirnov test was applied on data which revealed non-Gaussian distribution for cytokines $(P<0.05)$. Mean, SD, median was calculated for descriptive statistics. Median and Interquartile Range (IQR) were calculated for cytokine levels and ratios. MannWhitney U test was applied for comparison of PCAD patients and controls. Receiver operating characteristic curves (ROC) was constructed using MedCalc software in order to evaluate the diagnostic values including sensitivity, specificity, likelihood ratios and diagnostic odds ratios of cytokine mediators. Spearman Correlation test was applied to see the association between hS CRP and IL-18 and TNF alpha. A p-value of $<0.05$ was considered to be significant.

\section{Results}

A total of 196 subjects participated in this study comprising of 98 cases and 98 controls. The mean age of the cases and controls was $40.7 \pm 4.23$ and $35.1 \pm 7.55$ respectively. Baseline characteristic data of the subjects is shown in Table 1 . The PCAD patients were more frequently hypertensive, diabetic, and hyperlipidemia with a positive family history of PCAD.

ROC analysis of biomarkers in PCAD patients and controls revealed that Area under curve (AUC) and 95\% (CI) of hS CRP 0.936 (0.89-0.97) was the highest closely followed by IL-18: 0.853 (0.79-0.90). Area under curve (AUC) and 95\% (CI) of TNF alpha, IL-18/IL-10 ratio and IL-10 were 0.731 (0.659-0.796), 0.667 (0.592-0.737) and $0.574(0.497-0.649)$ respectively [Figure 1]. The diagnostic odds ratio of hS CRP was highest at a cutoff level of $3.18 \mathrm{mg} / \mathrm{L}$. The sensitivity and specificity of hS CRP at this cutoff for the diagnosis of PCAD was $86 \%$ and $91 \%$ respectively. 
Table 1: Demographic Characteristics of Patients and Controls.

\begin{tabular}{|c|c|c|c|}
\hline Parameters & Cases (n = 98) & $\begin{array}{c}\text { Controls (n } \\
\text { 98) }\end{array}$ & p-value \\
\hline Age (years) & $40 \pm 4.23$ & $35 \pm 7.55$ & 0.76 \\
\hline Gender(M/F) & $89 / 9$ & $93 / 5$ & 0.63 \\
\hline Weight (Kg) & $74.1 \pm 11.7 * *$ & $67.7 \pm 10.1$ & 0.0009 \\
\hline Height (m) & $1.68 \pm 0.06$ & $1.69 \pm 0.08$ & 0.85 \\
\hline BMI (Kg/ m²) & $26.32 \pm 3.7 * *$ & $23.6 \pm 3.5$ & $<0.002$ \\
\hline $\begin{array}{c}\text { Positive Diabetes } \\
\text { n (\%) }\end{array}$ & $36(37) * *$ & $3(4)$ & $<0.01$ \\
\hline $\begin{array}{c}\text { Positive } \\
\begin{array}{c}\text { Premature CAD } \\
\text { Family History } \\
\text { n (\%) }\end{array}\end{array}$ & $43(44) * *$ & $2(3)$ & $<0.01$ \\
\hline $\begin{array}{c}\text { Positive DM } \\
\text { family history } \\
\text { n (\%) }\end{array}$ & $36(37) * *$ & $11(15)$ & $<0.01$ \\
\hline $\begin{array}{c}\text { Smoker's n (\%) } \\
\text { Total Cholesterol } \\
\text { (mmol/ l) }\end{array}$ & $60(61)^{*}$ & $38(39)$ & $<0.05$ \\
\hline
\end{tabular}

PCAD: Premature Coronary Artery Disease; BMI: Body Mass Index; CAD: Coronary Artery Disease: DM: Diabetes Mellitus; $\mathrm{SD}=$ Standard Deviation Categorical variables were compared using $x^{2}$ test while continuous variables were compared using Welch's t-tests. ${ }^{*} p<0.05 ;{ }^{* *} p<0.01$.

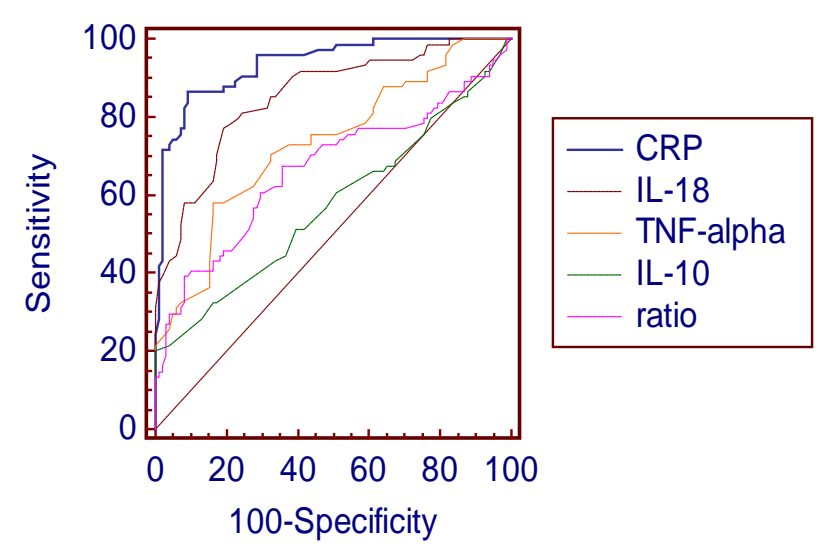

Figure 1: Receiver-Operator Characteristic (ROC) curve analyses of Interleukin-18 (IL- 18), Interleukin-10 (IL- 10), Tumor Necrosis Factoralpha (TNF alpha), IL- 18/ IL- 10 ratio and hS C-reactive protein (hS CRP) for diagnosis of premature coronary artery disease (PCAD) (n = 98).

The maximum sensitivity and specificity of IL-18, TNF alpha and IL-10 were seen at levels of $200 \mathrm{pg} / \mathrm{ml}, 4.6 \mathrm{pg} / \mathrm{ml}$ and $0.3 \mathrm{pg} /$ $\mathrm{ml}$ respectively, their DOR's were however lower than that of hS CRP as shown in [Table 2). Significant correlation was observed between hS CRP and IL-18 ( $\mathrm{r}=0.436, p<0.01)$ and hS CRP and TNF alpha $(r=0.287, p<0.05)$. The serum cytokine levels were significantly higher in cases as compared to the controls [Supplementary Table 1]. On performing logistic regression analysis after adjusting for the confounding variables IL-18, TNFalpha and hS-CRP remained highly significant $(p<0.01)$ while IL-
10 was moderately significant $(p<0.05)$ [Supplementary Table 2].

\section{Discussion}

The results of our study show that hS CRP have the best diagnostic ability out of all the cytokine mediators for the detection of PCAD. Patients with CAD had higher CRP levels than those without $\mathrm{CAD}$ and correlated with the severity of coronary atherosclerosis [11]. CRP is being quoted as the marker of disease activity as well as disease severity in cases of CAD [12]. This is because in the early stages of atherosclerosis it correlates extremely well with the rate of progression of atherosclerosis. hS CRP is the most predictive inflammatory marker for coronary artery disease [7]. Role of hS CRP in the risk stratification of CAD has also been shown by Peer et al. [13] more recently, Koc et al. [14] demonstrated that the levels of hS CRP were elevated in CAD when compared to controls regardless of the time of sampling and also had good diagnostic power for detection of stable CAD. Our study highlights the diagnostic performance of hS CRP in identifying premature CAD patients. Elzahwy et al. [15] demonstrated a strong correlation between hS CRP and coronary intima-media thickness which is an independent predictor of multi-level atherosclerosis and has high sensitivity and specificity However, Hung et al. [16] state that CRP provides only modest predictive value for future CAD Inflammatory biomarkers have immense potential in detection of coronary disease [17]. The importance of hS CRP in the diagnosis of PCAD emphasizes the need to determine its optimal cutoff value with the best sensitivity and specificity. The definitive cutoff values have still not been determined as the cut off value in our study was slightly higher and with much greater sensitivity and specificity as compared to a previous study [18].

Similarly, we observed that the sensitivity and specificity of IL-18 was also very high for the diagnosis of PCAD. Positive correlation has been shown between IL-18 and carotid intimamedia thickness and coronary plaque area [19]. This is probably because IL-18 over expression induces atheroma formation while the endogenous inhibitor of IL-18 slows plaque development [20]. Li et al. [21] demonstrated that IL-18 had AUC of 0.86 for detecting and predicting thin cap fibro-atheromas as confirmed on intravascular optical coherence tomography. On the other hand Martins et al. [22] found only marginal significance of IL-18 for prediction of CAD with or without adverse events.

TNF alpha and IL-18/IL-10 ratio showed moderate diagnostic performance for the diagnosis of PCAD. According to Branen et al. [23] TNF alpha is actively involved in the progression of atherosclerosis and its inhibition reduces atherosclerosis in mice. TNF alpha is being considered to be a sensitive and specific biomarker in the early diagnosis of metabolic syndrome which increases the risk to develop PCAD [24]. TNF alpha levels have shown significant association with the atherosclerotic burden consistently [25]. Chalikias et al. [26] demonstrated that an imbalance between the pro-and anti-inflammatory forces leads to plaque disruption and recurrent cardiovascular accidents making IL-18/IL-10 an independent predictor of adverse events 
Table 2: Diagnostic performance of hS CRP, IL-18, TNF alpha, IL-10 and IL-18/IL-10 ratio at different cut offs for diagnosis of PCAD.

\begin{tabular}{|c|c|c|c|c|c|c|c|}
\hline Biomarkers & SN (\%) & $95 \% \mathrm{CI}$ & SP (\%) & $95 \% \mathrm{CI}$ & LR+ & LR- & DOR \\
\hline hS- CRP $(\mathrm{mg} / \mathrm{L}) \geq 3.14$ & 85.14 & $75-92.3$ & 90.82 & $83.3-95.7$ & 9.27 & 0.16 & 58 \\
\hline$\geq 3.18^{*}$ & 86.49 & 76.5-93.3 & 90.82 & 83.3-95.7 & 9.42 & 0.15 & 62 \\
\hline$\geq 3.2$ & 86.49 & $76.5-93.3$ & 89.80 & $82.0-95.0$ & 8.48 & 0.15 & 57 \\
\hline IL-18 $(\mathrm{pg} / \mathrm{ml}) \geq 195$ & 70.27 & $58.5-80.3$ & 82.65 & 73.7-89.6 & 4.05 & 0.36 & 11 \\
\hline$\geq 200^{*}$ & 77.03 & $65.8-86.0$ & 80.61 & 71.4-87.9 & 3.97 & 0.28 & 14 \\
\hline$\geq 210$ & 78.38 & $67.3-87.1$ & 78.57 & $69.1-86.2$ & 3.66 & 0.28 & 13 \\
\hline TNF alpha $(\mathrm{pg} / \mathrm{ml}) \geq 4.5$ & 56.76 & $44.7-68.2$ & 83.67 & 74.8-90.4 & 3.48 & 0.52 & 6.7 \\
\hline$\geq 4.6^{*}$ & 58.11 & $46.1-69.5$ & 83.67 & 74.8-90.4 & 3.56 & 0.50 & 7.1 \\
\hline$\geq 4.8$ & 58.11 & $46.1-69.5$ & 80.61 & $71.4-87.9$ & 3.00 & 0.52 & 5.8 \\
\hline IL-18/IL-10 ratio $\geq 135.7$ & 66.22 & $54.3-76.8$ & 64.29 & $54.0-73.7$ & 1.85 & 0.53 & 3.5 \\
\hline$\geq 138.9^{*}$ & 67.57 & $55.7-78.0$ & 64.29 & $54.0-73.7$ & 1.89 & 0.50 & 3.8 \\
\hline$\geq 140$ & 67.57 & $55.7-78.0$ & 63.27 & $52.9-72.8$ & 1.84 & 0.51 & 3.6 \\
\hline IL-10 $(\mathrm{pg} / \mathrm{ml}) \geq 0.25$ & 13.51 & $6.7-23.5$ & 100.0 & $96.3-100$ & 5.5 & 0.86 & 6.4 \\
\hline$\geq 0.3^{*}$ & 20.27 & $11.8-31.2$ & 100.0 & $96.3-100$ & 5.35 & 0.80 & 6.7 \\
\hline$\geq 0.4$ & 21.62 & $12.9-32.7$ & 95.92 & 89.9-98.9 & 5.3 & 0.82 & 6.5 \\
\hline
\end{tabular}

hS- CRP: high sensitive C reactive protein, IL-18: Interleukin-18; IL-10: Interleukin-10; TNF alpha: Tumor Necrosis Factor-alpha; SN: Sensitivity; SP: Specificity; LR+: Positive likelihood ratio; LR-: Negative likelihood ratio; DOR: Diagnostic Odds Ratio; CI: Confidence Interval

in hospitalized coronary syndrome patients. The reason for their moderate diagnostic efficacy in our study is the possibility that acute cardiovascular event in our study population occurred over 2 months before the day of their angiography. So as the protective response comes into play after the acute event in the form of rise in IL-10 the IL-18/IL-10 ratio starts decreasing and TNF alpha degrades due to its short half life. IL-10 turned out to be highly specific at $0.3 \mathrm{pg} / \mathrm{ml}$ and may be used for correctly identifying the subjects not having PCAD. It can be employed as a rule out biochemical marker.

Significant positive correlation was observed between hS CRP and IL-18 highlighting the role and probable synergism of proinflammatory cytokine mediators in the diagnostic evaluation and pathogenesis of PCAD. Yamaoka-Tojo et al. [26] suggests that CRP induces the production of IL-18 further enhancing the pro-inflammatory component of the cytokine cascade. Moderate correlation was observed between hS CRP and TNF alpha also. This is in agreement with certain studies which suggest close correlation between hS CRP and TNF alpha [27]. One of the major strengths of our study is that we have independently established the reference interval of novel cytokine mediators in our study and have included angio-negative individuals as healthy controls thus allowing for a better comparison between patients and controls. It is also the first kind of its study to the best of our knowledge which has studied the diagnostic accuracy of proand anti-inflammatory cytokines in Premature Coronary artery disease patients who are young ( $<45$ years) and whose number is increasing alarmingly in Pakistan and South Asia. Moreover, we have seen that while IL-18 and hS CRP have high diagnostic performance for diagnosis of CAD and may be used in combination with clinical assessment for better therapeutic management. The major limitation of our study is its small sample size and the fact that we have included stable PCAD patients. We therefore recommend that future studies be carried out in multiple centers on larger patient populations and should also include acute coronary syndrome cases of PCAD for a better assessment of the diagnostic efficacy of cytokines in PCAD patients.

\section{Conclusion}

The study demonstrated that serum hS-CRP and IL-18 had more than $85 \%$ and $70 \%$ sensitivity respectively among the cytokines mediators for diagnosis of PCAD in the high risk group of patients. IL-18/IL-10 ratio revealed moderate diagnostic significance for PCAD. IL-10 had 100\% specificity highlighting its role in the exclusion of PCAD diagnosis in the cardiac clinic setup. These serum cytokines correlated well with the atherosclerosis burden. Thus, these potential cytokines can be used for triage of patients reported with chest pain and avoid the un-necessary costly angiography procedures in the healthy individual.

\section{Acknowledgement}

We wish to acknowledge the efforts of all the laboratory technicians who participated in the study.

\section{References}

1. Hansson GK, Robertson AK, Soderberg-Naucler C. Inflammation and atherosclerosis. Annu Rev Pathol. 2006; 1: 297-329.

2. Mills R, Bhatt DL. The Yin and Yang of arterial inflammation. J Am Coll Cardiol. 2004; 44(1): 50-52.

3. Sahar S, Dwarakanath RS, Reddy MA, Lanting L, Todorov I, Natarajan R. Angiotensin II Enhances Interleukin-18 Mediated Inflammatory Gene Expression In Vascular Smooth Muscle Cells -A Novel Cross-Talk in the Pathogenesis of Atherosclerosis. Circ Res. 2005; 96(10): 1064-1071.

4. Zhang C, Xu X, Potter BJ, Wang W, Kuo L, Michael L, et al. TNF alpha contributes to endothelial dysfunction in ischemia/reperfusion injury. Arterioscler Thromb VascBiol. 2006; 26(3): 475-480. 
5. Samnegård A, Hulthe J, Silveira A, Ericsson CG, Hamsten A, Eriksson P. Gender specific associations between matrix metalloproteinases and inflammatory markers in post myocardial infarction patients. Atherosclerosis. 2009; 202: 550-556. doi: 10.1016/j. atherosclerosis.2008.05.050.

6. Biswas S, Ghoshal PK, Mandal SC, Mandal N. Relation of anti- to Proinflammatory cytokine ratios with acute myocardial infarction. Korean J Intern Med. 2010; 25(1): 44-50. doi: 10.3904/kjim.2010.25.1.44.

7. Pearson TA, Mensah GA, Alexander RW, Anderson JL, Cannon RO $3^{\text {rd }}$, Criqui M, et al. Markers of inflammation and cardiovascular disease: application to clinical and public health practice: a statement for healthcare professionals from the Centers for Disease Control and Prevention and the American Heart Association. Circulation. 2003; 107(3): 499-511.

8. Sukhija R, Fahdi I, Garza L, Fink L, Scott M, Aude W, et al. Inflammatory markers, angiographic severity of coronary artery disease, and patient outcome. Am J Cardiol. 2007; 99(7): 879-884.

9. Pérez Fernández R, Kaski JC. [Interleukin-10 and Coronary Disease]. Rev Esp Cardiol. 2002; 55(7): 738-750.

10. Gensini GG. A more meaningful scoring system for determining the severity of coronary heart disease. Am J Cardiol 1983; 51(3): 606-607.

11. Momiyama Y, Ohmori R, Fayad ZA, Kihara T, Tanaka N, Kato R, et al. Associations between plasma C-reactive protein levels and the severity of coronary and aortic atherosclerosis. J Atheroscler Thromb. 2010; 17(5): 460-467.

12. Arroyo-Espliguero R, Avanzas P, Cosín-Sales J, Aldama G, Pizzi C, Kaski JC. C- protein elevation and disease activity in patients with coronary artery disease. Eur Heart J. 2004; 25(5): 401-408.

13. Peer A, Falkensammer G, Alber H, Kroiss A, Griesmacher A, Ulmer H et al. Limited utilities of $\mathrm{N}$-terminal pro B-type natriuretic peptide and other newer risk markers compared with traditional risk factors for prediction of significant angiographic lesions in stable coronary artery disease. Heart. 2004; 95(4): 297-303. doi: 10.1136/hrt.2008.145425.

14. Koc M, Karaarsalan O, Abali G, Batur MK. Variation in high sensitivity C reactive protein levels over 24 hours in patients with stable coronary artery disease. Tex Heart Inst J. 2010; 37(1): 42-48.

15. Elzahwy SS, Ewida II, Ismaeel MG, Hakeem SK. Value of carotid intimamedia thickness in estimating the spread of atherosclerosis and its relation to C-Reactive Protein. Heart Mirror J.2010; 4(1): 80-85

16. Hung J, Knuiman MW, Divitini ML, Langton PE, Chapman CL, Beilby J. C-reactive protein and interleukin-18 levels in relation to coronary heart disease: prospective cohort study from Busselton Western Australia. Heart Lung Circ. 2008; 17(2): 90-95.

17. Yayan J. Emerging families of biomarkers for coronary artery disease: inflammatory mediators. Vasc Health Risk Manag. 2013; 9: 435-456. doi: 10.2147/VHRM.S45704

18. Yilmaz N, Cicek HK, Celik A, Meram I, Kocabas R, Davutoglu V. Diagnostic value of homocysteine, C- reactive protein and bilirubin for coronary artery disease. Eastern Mediterr Health Journal. 2007; 13(3): 522-535.

19. Li C, Zhang XL, Liu H, Wang ZG, Yin KS. Association among plasma interleukin-18 levels, carotid intima- media thickness and severity of obstructive sleep apnea. Chin Medical J. 2009; 122(1): 24-29.

20. De Nooijer R, Von der Thusen JH, Verkleij CJN, Kuiper J, Jukema JW, van der Wall EE, et al. Over expression of IL-18 decreases intimal collagen content and promotes a vulnerable plaque phenotype in apolipoprotein-E-deficient mice. Arterioscler Thromb Vasc Biol. 2004; 24(12): 2313-2319.

21. Li QX, Fu QQ, Shi SW, Wang YF, XieJJ, Yu X, et al. Relationship between plasma inflammatory markers and plaque fibrous cap thickness determined by intravascular optical coherence tomography. Heart. 2010; 96: 196-201. doi: 10.1136/hrt.2009.175455.

22. Martins TB, Anderson JL, Muhlestein JB, Horne BD, CarlquistJF, Roberts WL, et al. Risk Factor Analysis of Plasma Cytokines in Patients with coronary artery disease by a multiplexed fluorescent immunoassay. Am J Clin Pathol. 2006; 125(6): 906-913.

23. Branen L, Hovgaard L, Nitulescu M, Bengtsson E, Nilsson J, Jovinge $S$. Inhibition of tumor necrosis factor- $\alpha$ reduces atherosclerosis in apolipoprotein E knockout mice. Arterioscler Thromb Vasc Biol. 2004; 24 (11): 2137-2142.

24. Gurrola-Díaz CM, Sánchez-Enriquez S, Oregon-Romero E, García-López PM, Garzón de la Mora P, Bastidas-Ramírez BE, et al. Establishment of a cut-point value of serum TNF- $\alpha$ levels in the metabolic syndrome. J Clin Lab Anal. 2009; 23(1): 1-69. doi: 10.1002/jcla.20289.

25. Battes LC, Cheng JM, Oemrawsingh RM, Boersma E, Garcia-Garcia HM, de Boer SP, et al. Circulating cytokines in relation to the extent and composition of coronary atherosclerosis: results from the ATHEROREMO-IVUS study. Atherosclerosis. 2014; 236(1): 18-24. doi: 10.1016/j.atherosclerosis.2014.06.010.

26. Chalikias GK, Tziakas DN, Kaski JC, Hatzinikolaou EI, Stakos DA, Tentes IK, et al. Interleukin-18: interleukin-10 ratio and in-hospital adverse events in patients with acute coronary syndrome. Atherosclerosis. 2005; 182(1): 135-143

27. Yamaoka-Tojo M, Tojo T, Masuda T, Machida Y, Kitano Y, Kurosawa T, et al. C-reactive protein-induced production of interleukin-18 in human endothelial cells: a mechanism of orchestrating cytokine cascade in acute coronary syndrome. Heart Vessels. 2003; 18(4): 183-187. 
Supplementary Table 1: Comparison of serum cytokine levels in Premature Coronary Artery Disease Patients and Controls.

\begin{tabular}{|c|c|c|c|c|}
\hline Parameters & B & SE & $\operatorname{Exp}(B)(95 \% C I)$ & Sig. \\
\hline IL- 18 (pg/ ml) & 0.020 & 0.003 & $1.020(1.004-1.019)^{* *}$ & 0.005 \\
\hline IL- $10(\mathrm{pg} / \mathrm{ml})$ & 0.310 & 0.182 & $1.062(0.842-2.037)^{*}$ & 0.04 \\
\hline TNF- alpha (pg/ ml) & 0.609 & 0.174 & $1.723(1.274-2.523)^{* *}$ & 0.001 \\
\hline hS- CRP (mg/ dl) & 0.450 & 0.124 & $1.561(1.253-2.145)^{* *}$ & 0.000 \\
\hline
\end{tabular}

IL: Interleukin; TNF-alpha: tumor necrosis factor-alpha; hS-CRP: High-sensitivity C - reactive protein; IQR: Interquartile range ${ }^{*} p<0.05 ;{ }^{* *} p<0.01$ applying Mann-Whitney $U$ test.

Supplementary Table 2: Multivariate Logistic Regression Analysis for Prediction of Premature Coronary artery disease.

\begin{tabular}{|c|c|c|c|c|}
\hline Parameters & B & SE & $\operatorname{Exp}(B)(95 \% C I)$ & Sig. \\
\hline IL- $18(\mathrm{pg} / \mathrm{ml})$ & 0.020 & 0.003 & $1.020(1.004-1.019)^{* *}$ & 0.005 \\
\hline IL- $10(\mathrm{pg} / \mathrm{ml})$ & 0.310 & 0.182 & $1.062(0.842-2.037)^{*}$ & 0.04 \\
\hline TNF- alpha (pg/ ml) & 0.609 & 0.174 & $1.723(1.274-2.523)^{* *}$ & 0.001 \\
\hline hS- CRP (mg/ dl) & 0.450 & 0.124 & $1.561(1.253-2.145)^{* *}$ & 0.000 \\
\hline
\end{tabular}

Logistic regression, adjusted for age, sex, smoking, BMI and diabetes, was performed for each group. SE: standard error; Exp (B): odds ratio; CI: Confidence interval; Sig: significance.

${ }^{* *} p<0.01 ;{ }^{*} p<0.05$ 